\title{
A newly modified esophagogastrostomy with a reliable angle of His by placing a gastric tube in the lower mediastinum in laparoscopy-assisted proximal gastrectomy
}

\author{
Atsushi Yasuda $\cdot$ Takushi Yasuda $\cdot$ Haruhiko Imamoto $\cdot$ Hiroaki Kato $\cdot$ \\ Kohei Nishiki · Mitsuru Iwama - Tomoki Makino • Osamu Shiraishi • \\ Masayuki Shinkai · Motohiro Imano · Hiroshi Furukawa · Kiyokata Okuno • \\ Hitoshi Shiozaki
}

Received: 31 January 2014/Accepted: 27 September 2014/Published online: 16 October 2014

(c) The International Gastric Cancer Association and The Japanese Gastric Cancer Association 2014

\begin{abstract}
Background An optimal reconstruction method for proximal gastrectomy $(\mathrm{PG})$ remains elusive. Esophagogastrostomy (EG) is technically simple but suffers from the disadvantage of gastroesophageal reflux. Jejunal interposition (JI) has a low rate of gastroesophageal reflux, but the procedure is more complicated, and delayed gastric emptying is a problem.

Methods We created a modified EG and have used the modified technique for PG since 2006. The procedure involves shaping the remnant stomach into a gastric conduit. The EG is performed high on the anterior wall, and the conduit is kept straight by applying a circular stapler inserted from the anterior wall of the antrum. The tip of the gastric conduit is then inserted into the lower mediastinum, creating a sharp angle of His. In this retrospective cohort study, the clinical and physiological outcomes were compared between 25 patients who underwent this procedure and 21 patients who underwent JI from 2001 to 2005 .

Results Laparoscopic procedures were performed more frequently, and residual food and bile reflux were less common in the EG group than in the JI group. No significant differences in remnant gastritis or reflux esophagitis were observed between the two groups. However, the late
\end{abstract}

A. Yasuda $(\bowtie) \cdot T$. Yasuda $\cdot H$. Imamoto $\cdot H$. Kato ·

K. Nishiki - M. Iwama · T. Makino · O. Shiraishi ·

M. Shinkai · H. Furukawa - K. Okuno · H. Shiozaki

Department of Surgery, Faculty of Medicine, Kinki University,

377-2 Ohno-higashi, Osaka-Sayama, Osaka 589-8511, Japan

e-mail: a-yasuda@surg.med.kindai.ac.jp

M. Imano

Cancer Center, Kinki University Hospital, 377-2 Ohno-higashi,

Osaka-Sayama, Osaka 589-8511, Japan complication of intestinal obstruction occurred only in the JI group.

Conclusions The modified EG technique has advantages over the JI technique because of its simplicity and low incidence of residual food and bile reflux. The next step would be to explore this technique further by a prospective multi-institutional study to confirm the reproducibility of its benefits. Miniabstract: The modified EG technique has advantages over the JI technique because of its simplicity, high rate of laparoscopy use, and low incidence of gastroesophageal reflux.

Keywords Proximal gastrectomy · Esophagogastrostomy · Gastric tube

\section{Introduction}

Advances in endoscopic technology and increased screening for gastric cancer over the past decade have increased the detection of early gastric cancer (EGC) [1,2]. Advances in endoscopic therapy have also enabled endoscopic resection of T1a mucosal cancer. It has been reported that compared with distal EGCs, a higher proportion of upper EGCs are submucosal tumors [3]. Therefore, some cases of upper EGC require surgery instead of endoscopic resection to achieve a cure. Recently, proximal gastrectomy (PG) has been widely performed for upper EGC to minimize surgical stress and preserve the function of the gastric remnant. Several studies have demonstrated that the outcome of PG is favorable, comparable to that of total gastrectomy (TG). PG is therefore a recognized alternative to $\mathrm{TG}$ for $\mathrm{EGC}$ in the upper stomach $[4,5]$.

However, the manner of reconstruction after PG remains controversial. Either esophagogastrostomy (EG) or jejunal 
interposition (JI) is usually performed. EG is technically simple; however, it is associated with a high incidence of postoperative gastroesophageal reflux. In contrast, JI, which was designed to reduce gastroesophageal reflux [6], is technically complicated and likely to induce delayed gastric emptying [7, 8]. Considering that these procedures are performed using a laparoscopic technique to minimize surgical stress, reconstruction with EG is likely advantageous in terms of simplicity; however, it needs to be modified to reduce gastroesophageal reflux.

Therefore, various measures such as shaping the remnant stomach into a gastric tube or using the lower esophageal sphincter-preserving technique $[9,10]$ have been employed. However, the reduction of gastroesophageal reflux remains unsatisfactory. We devised a new and simple EG reconstruction method to maintain a sharp angle of His by placing the tip of the remnant stomach shaped like a gastric tube into the lower mediastinum, thereby preventing regurgitation. We have applied this reconstruction method for laparoscopyassisted PG (LAPG) since 2006. In this retrospective cohort study, we reviewed the clinical outcomes of this technique and assessed its safety and physiological advantage.

\section{Patients and methods}

A total of 46 patients with EGC in the upper third of the stomach underwent PG at our institution between January 2001 and December 2011. JI reconstruction was performed in 21 patients up until 2006 (JI group), and modified EG reconstruction has been performed in 25 patients since 2006 (EG group). All the patients had stage IA or IB gastric cancer preoperatively confirmed by gastroendoscopy and computed tomography (CT) (Japanese Classification of Gastric Carcinoma, version 14) [11]. The indication for PG at our institution was particularly limited to cT1 EGC located in the upper third of the stomach.

The postoperative physiological function was evaluated in the EG group by a fluoroscopic study with gastrographin on postoperative day 5 , which assessed passage in an upright position, esophageal reflux in a head-down position, and the presence of periodic peristalsis of the lower gastric conduit. Postoperative complications were evaluated according to the Clavien-Dindo classification [12], and grade II or higher was considered significant. Early and late complications were defined as those that occurred within and after 1 month after surgery, respectively. Postoperative stenosis was defined as cases that required endoscopic balloon dilatation even just once. The states of the remnant stomach and reflux esophagitis were evaluated by endoscopic examination from 1-year follow-up according to the residual food, gastritis, and bile reflux (RGB) classification and the Los Angeles classification, respectively. The RGB classification has six grades (0-5) for the degree of residual food and gastritis and three grades (0-2) for the degree of bile reflux [13].

Surgical techniques

In both the EG and GI groups, following laparotomy with an upper midline incision or a laparoccopic approach, D1+ lymph node dissection was performed according to the Japanese Gastric Cancer Treatment Guideline 2010 (3rd edition) [14]. The hepatic and celiac branches of the vagus nerve were preserved. Following lymphadenectomy, the abdominal esophagus was transected, and the anvil of the circular stapler device (CDH, $25 \mathrm{~mm}$, Ethicon Ltd., Edinburgh, UK) was inserted into the esophagus and placed with a purse-string suture using the purse-string instrument (PSI) (Fig. 1a-d). Following this, the stomach was pulled out from the abdominal cavity through the upper midline incision and divided using a linear stapler (TLC75, Ethicon Ltd.) at the line just below the marking clip, and the cut end was inverted with a Lembert suture using 3-0 Vicryl (Ethicon Ltd.). Reconstruction was performed after confirming the negative surgical margin by intraoperative rapid diagnosis with a frozen section. Pyloric manipulation was not routinely performed in all cases.

\section{Basic procedures for the modified EG}

After trimming the esophagus for resection, the esophageal hiatus was dilated by resecting the phrenoesophageal ligament and the infradiaphragmatic fascia around the hiatus, following which blunt dissection of the lower mediastinum to $4-5 \mathrm{~cm}$ above the hiatus was performed, thereby creating space to insert the tip of the remnant stomach into the mediastinum. This was followed by transection of the esophagus and fixation of the anvil at the esophageal stump.

The gastric body was diagonally divided from the lower portion of the lesser curvature toward the upper portion of the greater curvature using a linear stapler to create the gastric conduit (20 cm long, 5-7 cm wide) (Fig. 2a). A gastrotomy was made in the lower anterior wall of the conduit at $4-5 \mathrm{~cm}$ from the pylorus for insertion of the circular stapler device ( $\mathrm{CDH}, 25 \mathrm{~mm})$. The $\mathrm{CDH}$ was then inserted into the gastric conduit in a retrograde fashion, and EG was performed at the appropriate position to keep the conduit straight in the higher anterior wall, usually $5-7 \mathrm{~cm}$ distal to the tip (Fig. 2b). The gastrotomy was closed using a hand-sewn technique with 3-0 Vicryl. Finally, the tip of the gastric conduit corresponding to the proximal part above the level of the EG, which is called the pseudofornix of the gastric remnant, was inserted into the lower mediastinal space and anchored with the diaphragm by a few stitches as well as the fornix, creating the new cardiac notch (angle of His) (Fig. 2c). 

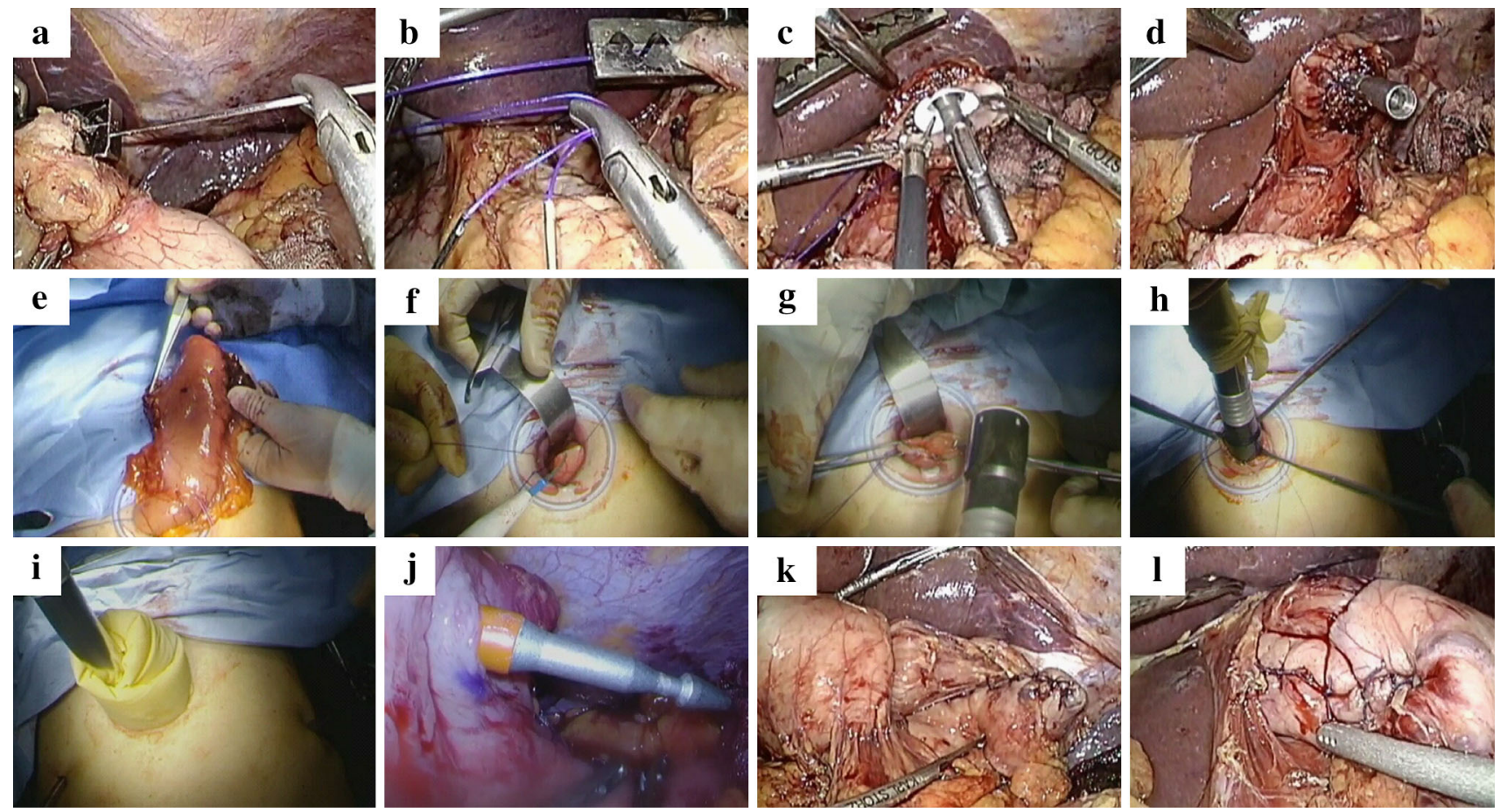

Fig. 1 Insert a PSI into the abdomen through the glove. Apply the instrument on the esophagus along the scheduled incisional line before getting a straight needle through $(\mathbf{a}, \mathbf{b})$. Insert the anvil into the esophagus and anchor it with extra- or intracorporal ligation $(\mathbf{c}, \mathbf{d})$. Mark the scheduled anastomotic site on the gastric conduit (e). Place two sutures on the anterior wall of the antrum. Make a small incision between the sutures for introduction of the $\mathrm{CDH}$ device into the conduit $(\mathbf{f}-\mathbf{h})$. Insufflate the abdomen with the glove-port technique

(i). Thrust out the shaft of the $\mathrm{CDH}$ device through the gastric wall inside the abdomen (j). Fold the pseudo-fornix downward and be careful not to involve the surrounding tissue before firing the $\mathrm{CDH}$ stapler (k). After firing, pull out the pseudo-fornix from the lesser curvature, and complete the reconstruction procedures by delivering the tip into the mediastinum. Placement of the tip in the mediastinum is expected to keep the tip behind the esophagus (I)
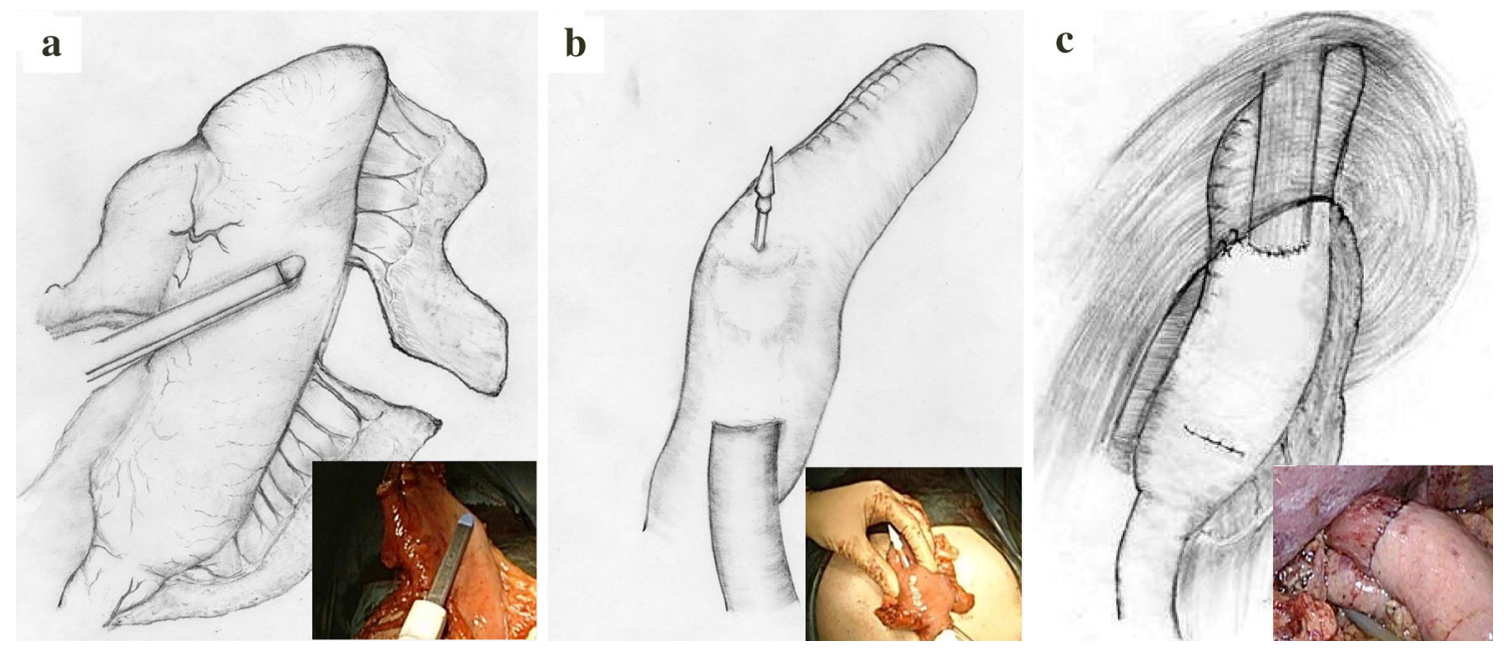

Fig. 2 The EG procedure. a Incision of the stomach and creation of the gastric conduit. $\mathbf{b}$ EG at the anterior wall of the stomach. $\mathbf{c}$ Completion of the EG. The pseudo-fornix can be seen at the back of the EG

\section{Additional tips for the modified EG by the laparoscopic approach}

The laparoscopic approach has been employed for PG with modified EG since 2007. Five-port access, comprising a 10-mm umbilical port, 5-mm right lower quadrant port, 10-mm left upper quadrant port, 5-mm left lower quadrant port, and $10-\mathrm{mm}$ epigastric port, with an upper midline incision of $3.5 \mathrm{~cm}$, was used. All surgical techniques for PG and EG reconstruction were 
laparoscopically performed using the same procedures as those for open surgery. Dilatations of the esophageal hiatus and lower mediastinum were easily conducted in a laparoscopic fashion using an energy device or by blunt dissection using forceps because of its loose connective tissue. PG and creation of the gastric conduit were performed as described previously because the stomach can be pulled out from the abdominal cavity through the upper midline incision.

In low-weight patients with thin abdominal walls, insertion of the anvil and EG using the $\mathrm{CDH}$ were also performed by direct maneuvers through this small incision with the assistance of laparoscopic vision.

On the contrary, in obese patients with thick abdominal walls, $3.5-\mathrm{cm}$ incisions were too small to allow the entry of the conduit with the $\mathrm{CDH}$ into the abdominal cavity. Therefore, we inserted the $\mathrm{CDH}$ into the gastric conduit inside the abdominal cavity, assisted by the laparoscope. The procedures are described as follows:

1. The scheduled anastomotic site on the gastric conduit outside the abdominal cavity was marked before replacing it in the cavity (Fig. 1e).

2. Two sutures on the anterior wall of the antrum were placed, and a small incision between the sutures (Fig. 1f) was made for the introduction of the $\mathrm{CDH}$ into the conduit (Fig. 1g, h).

3. The abdomen was insufflated with the glove port technique (Fig. 1i). The shaft of the $\mathrm{CDH}$ was thrust out through the gastric wall inside the abdomen (Fig. 1j).

4. The pseudo-fornix was folded downward, and care was taken not to involve the surrounding tissue before firing the $\mathrm{CDH}$ device (Fig. 1k). After firing, the pseudo-fornix from the lesser curvature was pulled out, and the reconstruction procedures were completed by delivering the tip into the mediastinum (Fig. 11).

The entry hole of the $\mathrm{CDH}$ at the anterior wall of the antrum was directly closed using the hand-sewn method through a small incision, and the pseudo-fornix was placed into the mediastinal space using the laparoscopic technique.

\section{JI reconstruction}

After the jejunal segment had been adjusted to a proper length of approximately $15 \mathrm{~cm}$ and the proximal end of the jejunum anastomosed to the esophagus using the CDH (ILS 25, Ethicon), the distal end was anastomosed to the gastric remnant in the anterior wall using manual sutures. The same reconstruction maneuver performed in the open laparotomy surgery described above was also performed in the laparoscopic surgery, referring to Ikeda et al. [15].

Follow-up

Patients were followed as outpatients every 3-6 months for the first 5 years and annually thereafter. Endoscopy and CT of the chest and abdomen were performed annually.

Statistical analysis

Categorical data were compared using the $\chi^{2}$ test or Fischer's exact test. Other variables were compared using the Mann-Whitney $U$ test. $p$ values $<0.05$ were considered statistically significant. All analyses were performed with the JMP statistical software package (version 9, SAS Institute, Cary, NC, USA).

\section{Results}

\section{Patient characteristics}

The clinicopathological characteristics of the patients are summarized in Table 1. The mean age of the EG group was significantly higher than that of the JI group, while there was no significant difference in sex, pathological stage, histological type, operation time, blood loss, and extent of lymphadenectomy between the two groups. However, the proportions of laparoscopic surgery and postoperative hospital stay were significantly higher $(p<0.001)$ and shorter by approximately 10 days $(p=0.031)$, respectively, in the EG group than in the JI group (Table 1). Proton pump inhibitors (PPIs) or camostat mesilate had been prescribed for all the patients in the EG group and 14 patients in the JI group after PG $(p=0.002)$.

Early postoperative complications

There was no significant difference in early postoperative complications between the EG and JI groups. In addition, no pulmonary complications were observed throughout this study (Table 2A).

Postoperative physiological function

In the EG group, the postoperative gastrographin study showed smooth passage of the medium in an upright position (Fig. 3a), and reflux was not observed even in the head-down position (Fig. 3b). Serial gastrography revealed the presence of periodic peristalsis of the lower gastric conduit (Fig. 3c-f). 
Table 1 Patient characteristics

\begin{tabular}{|c|c|c|c|c|}
\hline & & $\begin{array}{l}\text { EG group } \\
(n=25)\end{array}$ & $\begin{array}{l}\text { JI group } \\
(n=21)\end{array}$ & $P$ value \\
\hline Age (years) & & $71.6 \pm 8.6^{\mathrm{a}}$ & $61.0 \pm 9.5^{\mathrm{a}}$ & $<0.001$ \\
\hline Sex & Male/female & $18 / 7$ & $13 / 8$ & 0.538 \\
\hline pStage & IA/IB/IIA/IIB & $19 / 5 / 0 / 1$ & $19 / 1 / 1 / 0$ & 0.100 \\
\hline Histological type & $\begin{array}{l}\text { Differentiated/ } \\
\text { diffuse }\end{array}$ & $10 / 15$ & $13 / 8$ & 0.735 \\
\hline \multicolumn{5}{|l|}{ Surgical procedure } \\
\hline Open/laparoscopic & & $5 / 20$ & $16 / 5$ & $<0.001$ \\
\hline Operation time (min) & & $286.4 \pm 54.3^{\mathrm{a}}$ & $268.8 \pm 59.6^{\mathrm{a}}$ & 0.160 \\
\hline Blood loss (ml) & & $294.2 \pm 334.5^{\mathrm{a}}$ & $307.4 \pm 264.8^{\mathrm{a}}$ & 0.078 \\
\hline Lymph node dissection & & D1+ & D1+ & - \\
\hline Postoperative hospital stay (days) & & $18.6 \pm 5.6^{\mathrm{a}}$ & $29.4 \pm 19.8^{\mathrm{a}}$ & 0.031 \\
\hline $\begin{array}{l}\text { Postoperative antacid therapy (PPIs or } \\
\text { camostat mesilate) }\end{array}$ & $( \pm)$ & $25 / 0^{\mathrm{a}}$ & $14 / 7^{\mathrm{a}}$ & 0.002 \\
\hline
\end{tabular}

Table 2 Postoperative complications after proximal gastrectomy

\begin{tabular}{|c|c|c|c|}
\hline Complication & EG group $(n=25)$ & JI group $(n=21)$ & $p$ value \\
\hline \multicolumn{4}{|c|}{ A. Early postoperative complications } \\
\hline Leakage & 0 & 2 & 0.203 \\
\hline Bleeding & 0 & 1 & 0.457 \\
\hline Stenosis & 0 & 3 & 0.088 \\
\hline Pulmonary related & 0 & 0 & - \\
\hline Intraabdominal abscess & 1 & 1 & 0.710 \\
\hline Wound infection & 3 & 2 & 0.767 \\
\hline Total & $4(16 \%)$ & $6(28.6 \%)$ & 0.251 \\
\hline Complication & EG group $(n=23)$ & JI group $(n=20)$ & $p$ value \\
\hline \multicolumn{4}{|c|}{ B. Late postoperative complications } \\
\hline Anastomotic stenosis & 5 & 2 & 0.298 \\
\hline Reflux syndrome & 1 & 1 & 0.919 \\
\hline Intestinal obstruction & 0 & 4 & 0.024 \\
\hline Total & $5(21.7 \%)$ & $5(25.0 \%)$ & 0.801 \\
\hline
\end{tabular}

$E G$ esophagogastrostomy, $J I$ jejunal interposition

Evaluation of mucosal state and physiological function

Excluding 7 patients ( 6 were transferred to other hospitals, 1 died of peritonitis carcinomatosa within 1 year of surgery), the remaining 39 patients were followed regularly (22 patients in the EG group and 17 patients in the JI group).

Food residue in the gastric remnant and bile reflux of grade 1 or higher were more common in the JI group than in the EG group ( $p=0.009$ and $p=0.041$, respectively), while no significant difference was observed in the grade of remnant gastritis between the two groups $(p=0.234)$ (Table 3). Occurrence of reflux esophagitis was also similar between the groups $(p=0.416)$ (Table 4). However, severe esophagitis of grade $\mathrm{C}$ or $\mathrm{D}$ was only observed in two patients in the EG group: one with gibbus deformity and the other who underwent reanastomosis using a handsewn technique because of failure of the CHD device. Excluding these 2 patients, the distribution of esophagitis grade was comparable between the 2 groups, and 13 (59 \%) of the 22 patients with grade $\mathrm{N}$ were observed even in the EG group. To exclude the beneficial effects of PPIs or camostat mesilate, the same comparison for each item was performed by focusing on patients on these drugs. All the results showed similar tendencies (Tables 3, 4). Routine endoscopic examinations performed 1 year postoperatively showed that the gross anatomical structure of the pseudo-fornix remained intact, as had been designed during surgery in all cases. A typical endoscopic finding is shown in Fig. 3g, h. 

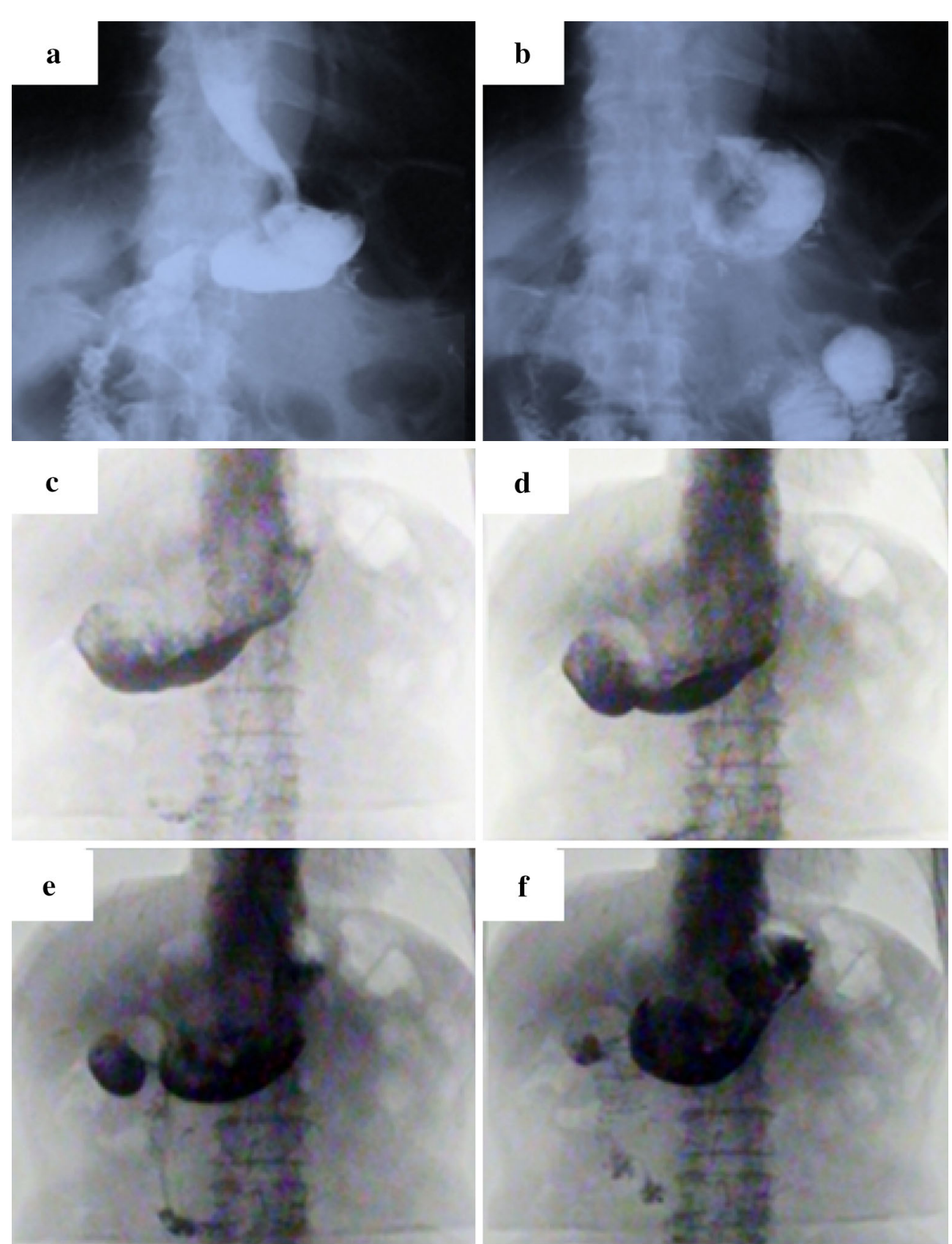

d
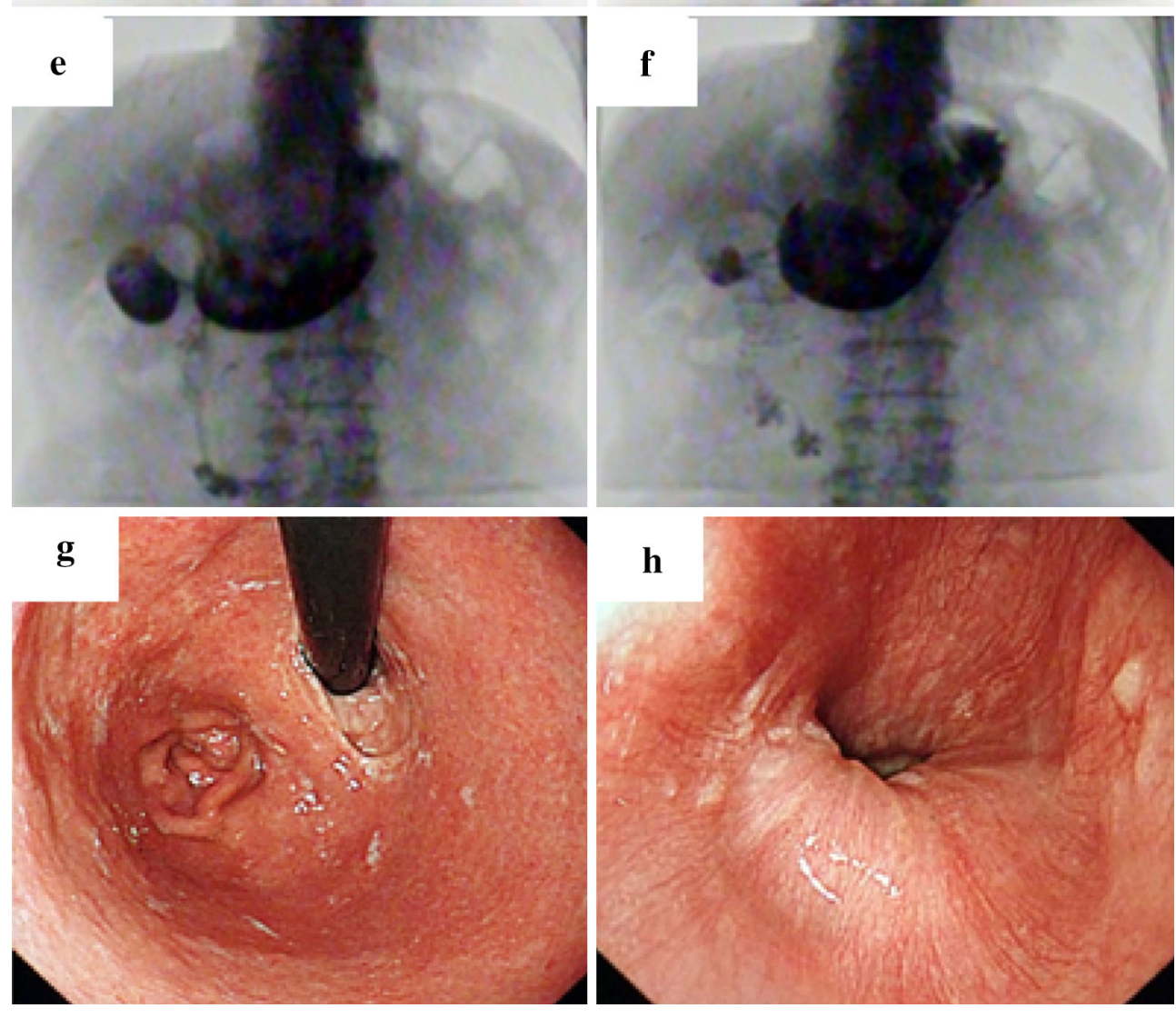
4Fig. 3 Postoperative upper gastrointestinal series. a Upright position. b Head-down position. c-f Peristalsis of the lower gastric conduit. One-year postoperative endoscopic examination findings in the EG group. $\mathrm{g}$ A pseudo-fornix formed in the gastric remnant (left side of the $\mathbf{e}-\mathbf{g}$ orifice). $\mathbf{h}$ No esophagitis in the lower esophagus

Table 3 Residual food, gastritis, and bile reflux score

\begin{tabular}{cllll}
\hline Grade & EG group $(n=22)$ & JI group $(n=17)$ & $p$ value & \\
\hline Residue & & & & \\
0 & $18(18)$ & $7(4)$ & $0.009^{*}$ & $(0.009)^{\#}$ \\
1 & $4(4)$ & 0 & & \\
2 & 0 & $2(2)$ & & \\
3 & 0 & $3(2)$ & & \\
4 & 0 & $5(3)$ & & \\
Gastritis & & & \\
0 & $6(6)$ & $2(0)$ & & \\
1 & $15(15)$ & $11(8)$ & & \\
2 & $1(1)$ & $4(3)$ & & \\
3 & 0 & 0 & & \\
Bile reflux & & & \\
0 & $15(15)$ & $6(3)$ & & \\
1 & $7(7)$ & $11(8)$ & & \\
\hline
\end{tabular}

Numbers of patients who had taken either proton pump inhibitors or camostat mesilate are shown in parentheses

$E G$ esophagogastrostomy, $J I$ jejunal interposition

* Score 0 versus 1 or more

\# Comparison of medicated patients between the groups

Table 4 Modified Los Angeles classification

\begin{tabular}{lllll}
\hline Grade & EG group $(n=22)$ & JI group $(n=17)$ & $p$ value & \\
\hline $\mathrm{N}$ & $13(13)$ & $10(6)$ & 0.416 & $(0.543)^{\#}$ \\
$\mathrm{M}$ & $1(1)$ & $3(2)$ & & \\
$\mathrm{A}$ & $5(5)$ & $4(3)$ & & \\
$\mathrm{B}$ & $1(1)$ & 0 & \\
C or D & $2(2)$ & 0 & & \\
\hline
\end{tabular}

Numbers of patients who had taken either proton pump inhibitors or camostat mesilate are shown in parentheses

$E G$ esophagogastrostomy, $J I$ jejunal interposition

\# Comparison of medicated patients between both groups

Late postoperative complications

There was no significant difference in the incidence of total late postoperative complications, anastomotic stenosis, and reflux syndrome between the two groups. Small bowel obstruction was observed only in the JI group (Table 2b).

\section{Discussion}

In the past decade, PG has been performed for upper EGC with excellent oncological outcomes [16, 17]. However, PG involves destruction of the angle of His and deformation and downsizing of the stomach. PG has a high risk of postoperative gastroesophageal reflux and food stagnation, both of which markedly decrease the quality of life of patients. Laparoscopic surgery has emerged as an option for the surgical treatment of EGC, including PG. Laparoscopic reconstruction and improvement of reconstruction methods in PG to prevent regurgitation of gastric contents and facilitate their discharge into the duodenum are important issues.

Some studies have shown that JI reconstruction shows favorable postoperative results and diet tolerance [16, 17] and that the addition of pyloroplasty allows for good dietary intake, early recovery of body weight, and smooth gastric emptying [18]. We therefore selected JI before 2005 , but found the procedure to be complicated since it requires a greater number of anastomoses. In addition, JI resulted in four cases of readmission because of bowel obstruction. Thus, we developed a modified version of EG, which, despite requiring some additional procedures to pursue improvements in postoperative quality of life, remains technically simple.

With regard to EG reconstruction, Adachi et al. [19] and Shiraishi et al. [20] performed EG with a narrow gastric tube and reported it to be a safe and simple procedure with benefits such as a shorter operation time, faster recovery, and lower hospital expenses compared with JI and an equally low incidence of reflux esophagitis despite an endto-end anastomosis. However, regurgitation when lying down cannot be avoided. Meanwhile, Ichikawa et al. [21] reported that EG was performed in an end-to-side fashion using a narrow gastric tube laparoscopically, resulting in favorable clinical outcomes, including a low incidence of reflux esophagitis and preservation of the physiological function of the remnant stomach. However, the length of the newly created pseudo-fornix was less than $3 \mathrm{~cm}$, which seemed to be inadequate to function as an angle of His for the prevention of reflux.

Against these backgrounds, we modified the conventional EG in 2006 by focusing on the prevention of gastroesophageal reflux and gastrointestinal stasis, and we have performed this procedure using a laparoscopy-assisted approach since 2007. The uniqueness and advantages of this technique are provided below.

First, a gastric remnant is shaped into a 5-7-cm-wide, narrow gastric tube because a low compliance of the gastric remnant wall is likely advantageous in preventing regurgitation and facilitating drainage into the duodenum. 
Bemelman et al. [22] examined the incidence of delayed gastric emptying by the size of the gastric remnant (the whole stomach, distal two-thirds of the stomach, and tubulized stomach) in patients who underwent esophagectomy and reported a significantly high incidence in the whole stomach group. Gastric emptying mainly depends on gravitational forces and the buildup of intraluminal gastric pressure; therefore, a small gastric tube leads to a rapid increase in gastric pressure when the stomach is filled, thereby facilitating gastric emptying [22, 23]. In addition, because the newly created pseudo-fornix is placed into the lower mediastinum in our procedure, the gastric remnant with a high compliance is susceptible to intrathoracic negative pressure; therefore, the change in intragastric pressure along with breathing likely induces duodenogastric reflux [24]. Animal experiments also indicated that the smaller the initial volume of the gastric remnant, the lower the compliance of the gastric wall is [24, 25]. Consequently, a narrow gastric tube has an advantage in gastric emptying.

Second, the $\mathrm{CDH}$ is inserted through the gastrotomy at the anterior wall of the antrum in a retrograde manner. Considering that gravity and increased intragastric pressure move the gastric contents into the duodenum, it is crucial to keep the gastric remnant straight and prevent it from hanging down. In this regard, our method is advantageous because it is easy to adjust the site of EG and maintain a straight greater curvature line.

Third, the EG is placed on the anterior wall of the gastric tube. This technique enables the formation of a pseudofornix on the back of the lower esophagus. Fourth, the tip of the gastric conduit, the pseudo-fornix, is inserted into the lower mediastinal space after dilating the esophageal hiatus. The third and fourth procedures ensure that a sharp angle of His is formed and that the positional relationship is maintained in the space surrounded by the heart, pleura, aorta, and vertebra. In addition, because the pseudo-fornix behind the esophagus could function as a reservoir, the gastroesophageal reflux that often occurs when patients lie down [26] could be reduced. If the pseudo-fornix is not placed in the lower mediastinum, the esophageal hiatus of the diaphragm may disrupt the sharp angle of His by pushing away the tip of the gastric conduit laterally, even if the pseudo-fornix is sufficiently long. However, a short pseudo-fornix would be insufficient to prevent gastroesophageal reflux.

A postoperative upper GI series showed that there was good passage to the duodenum, an absence of esophageal reflux even in a head-down position, and periodic peristalsis of the lower gastric conduit due to preservation of the hepatic and pyloric branches of the vagus nerve. Postoperative endoscopic surveillance also verified that the grade of esophagitis was almost equal between the two groups, while food residue in the remnant stomach and bile reflux scores were significantly lower in the EG group than in the JI group [grade $0 ; 15 / 22(68.2 \%)$ and 6/17 (35.3\%), respectively]. In particular, bile reflux was commonly observed in the JI group, although the hepatic and pyloric branches of the vagus nerve were preserved in both the groups.

We were unable to clearly identify etiology of the three cases of severe postoperative reflux esophagitis in the GE group at this time and could not completely deny the possibility of their association with our surgical procedure.

JI appeared to effectively function to reduce gastroesophageal reflux; however, hypoperistalsis due to denervation and a high compliance of the jejunal wall was likely to function the other way with regard to duodenogastric reflux, suggesting that the intraluminal negative pressure in the gastric remnant and the interposed jejunum may be yielded by the negative intraabdominal pressure with inspiration and cause bile reflux [24].

Despite no difference being observed in the incidence of perioperative complications between the two groups, leakage and anastomotic stenosis resulting in a longer hospital stay occurred only in the JI group. Furthermore, bowel obstruction as a late complication occurred only in the JI group, although it may not be appropriate to overemphasize the association between JI and bowel obstruction based on a small case series.

The limitation of this study is that this study prospectively compared the JI group as the historical control group with the EG group, and it was a small-scale, non-randomized, single-institute study. In addition, because the surgical approaches, open or laparoscopic surgery, were not regulated in this study, the applicability of each reconstruction method to laparoscopic surgery cannot be accurately evaluated.

In conclusion, our modified EG reconstruction method was technically feasible even when performed laparoscopically. In addition, the formation of a pseudo-fornix and optimal angle of His appeared to reduce bile reflux, while the gastric remnant in the form of a narrow gastric tube with low compliance resulted in decreased food residue. In the future, our method could be evaluated in a prospective study with patient-reported outcome as one of endpoints, pending pilot studies in other institutions to ensure its reproducibility.

\section{References}

1. Kong SH, Park DJ, Lee HJ, Jung HC, Lee KU, Choe KJ, Yang HK. Clinicopathologic features of asymptomatic gastric adenocarcinoma patients in Korea. Jpn J Clin Oncol. 2004;34(1):1-7. 
2. Kunisaki C, Ishino J, Nakajima $S$, Motohashi H, Akiyama $H$, Nomura M, Matsuda G, Otsuka Y, Ono HA, Shimada H. Outcomes of mass screening for gastric carcinoma. Ann Surg Oncol. 2006;13(2):221-8 Epub 2006 Jan 17.

3. Okabayashi T, Gotoda T, Kondo H, Inui T, Ono H, Saito D, Yoshida S, Sasako M, Shimoda T. Early carcinoma of the gastric cardia in Japan: is it different from that in the West? Cancer. 2000;89(12):2555-9.

4. Shiraishi N, Adachi Y, Kitano K, Kakisako K, Inomata M, Yasuda K. Clinical outcome of proximal versus total gastrectomy for proximal gastric cancer. World J Surg. 2002;26:1150-4.

5. Furukawa $H$, Hiratsuka $M$, Imaoka $S$, Ishikawa $O$, Kabuto $T$, Sasaki Y, Kameyama M, Ohigashi H, Nakano H, Yasuda T. Limited surgery for early gastric cancer in cardia. Ann Surg Oncol. 1998;5(4):338-41.

6. Hsu CP, Chen CY, Hseih YH, Hsia JY, Shai SE, Kao CH. Esophageal reflux after total or proximal gastrectomy in patients with adenocarcinoma of the gastric cardia. Am J Gastroenterol. 1997;92(8):1347-50.

7. Katsube T, Konnno S, Hamaguchi K, et al. Complications after proximal gastrectomy with jejunal pouch interposition : report of a case. Eur J Surg Oncol. 2005;31(9):1036-8.

8. Ueno M, Iwahashi M, Nakamori M, et al. Complication of jejunal pouch interposition after proximal gastrectomy : case report. Hepatogastroenterology. 2004;51(57):916-8.

9. Shiraisi Norio, et al. Gastric tube reconstruction prevented esophageal reflex after proximal gastrectomy. Gastric Cancer. 1998;1:78-9.

10. Kim DJ, Lee JH, Kim W. Lower esophageal sphincter-preserving laparoscopy-assisted proximal gastrectomy in patients with early gastric cancer: a method for the prevention of reflux esophagitis. Gastric Cancer. 2012 Oct 13. [Epub ahead of print].

11. Association Japanese Gastric Cancer. Japanese classification of gastric carcinoma: 3rd English edition. Gastric Cancer. 2011;14: 101-12.

12. Dindo D, Demartines N, Clavien PA. Classification of surgical complications: a new proposal with evaluation in a cohort of 6336 patients and results of a survey. Ann Surg. 2004;240: 205-13.

13. Kubo M, Sasako M, Gotoda T, et al. Endoscopic evaluation of the remnant stomach after gastrectomy: proposal for a new classification. Gastric Cancer. 2002;5:83-9.
14. Association Japanese Gastric Cancer. Japanese gastric cancer treatment guidelines 2010 (ver. 3). Gastric Cancer. 2011;14: $113-23$.

15. Ikeda Y, Sasaki Y, Niimi M, Kan S, Takami H, Kodaira S. Handassisted laparoscopic proximal gastrectomy with jejunal interposition and lymphadenectomy. J Am Coll Surg. 2002;195(4): $578-81$.

16. Iwata T, Kurita N, Ikemoto T, Nishioka M, Andoh T, Shimada M. Evaluation of reconstruction after proximal gastrectomy: prospective comparative study of jejunal interposition and jejunal pouch interposition. Hepatogastroenterology. 2006;53(68):301-3.

17. Takeshita K, Sekita Y, Tani M. Medium- and long-term results of jejunal pouch reconstruction after a total and proximal gastrectomy. Surg Today. 2007;37(9):754-61.

18. Nakane $Y$, Michiura T, Inoue K, Sato M, Nakai K, Ioka M, Yamamichi K. Role of pyloroplasty after proximal gastrectomy for cancer. Hepatogastroenterology. 2004; 51:1867-71.

19. Adachi Y, Inoue T, Hagino Y, Shiraishi N, Shimoda K, Kitano S. Surgical results of proximal gastrectomy for early-stage gastric cancer: jejunal interposition and gastric tube reconstruction. Gastric Cancer. 1999;2:40-50.

20. Shiraishi N, Adachi Y, Kitano K, Kakisako K, Inomata M, Yasuda K. Clinical outcome of proximal versus total gastrectomy for proximal gastric cancer. World J Surg. 2002;26:1150-4.

21. Ichikawa D, Komatsu S, Okamoto K, Shiozaki A, Fujiwara H, Otsuji E. Esophagogastrostomy using a circular stapler in laparoscopy-assisted proximal gastrectomy with an incision in the left abdomen. Langenbecks Arch Surg. 2012;397(1):57-62.

22. Bemelman WA, et al. Delayed postoperative emptying after esophageal resection is dependent on the size of the gastric substitute. J Am Coll Surg. 1995;180(4):461-4.

23. D'Journo XB, Martin J, Ferraro P, Duranceau A. The esophageal remnant after gastric interposition. Dis Esophagus. 2008;21: 377-88.

24. Bemelman WA, Verburg J, Brummelkamp WH, Klopper PJ. A physical model of the intrathoracic stomach. Am J Physiol. 1988;254:G168-75.

25. Stadaas J, Aune S. Intragastric pressure/volume relationship before and after vagotomy. Acta Chir. Scand. 1970;136:611-5.

26. Demeester TR, Johnson LF, Joseph GJ, Toscano MS, Hall AW, Skinner DB. Patterns of gastroesophageal reflux in health and disease. Ann Surg. 1976;184:459-70. 